\title{
Enhancing Learning Management Systems Utility for Blind Students: A Task-oriented, User- Centered, Multi-Method Evaluation Technique
}

\author{
Rakesh Babu \\ School of Information Studies, \\ University of Wisconsin-Milwaukee, Milwaukee, WI, USA \\ babu@uwm.edu \\ Rahul Singh \\ Bryan School of Business and Economics, \\ The University of North Carolina at Greensboro, NC, USA
}

rahul@uncg.edu

\section{Executive Summary}

This paper presents a novel task-oriented, user-centered, multi-method evaluation (TUME) technique and shows how it is useful in providing a more complete, practical and solution-oriented assessment of the accessibility and usability of Learning Management Systems (LMS) for blind and visually impaired (BVI) students. Novel components of TUME include a purposeful integration of a multi-theoretic foundation and multiple methods to accurately identify users' accessibility and usability problems in Web interaction and identify design problems and solutions to ensure technical feasibility of recommendations. The problems identified by TUME remain hidden from extant evaluation methods - therefore, these problems remain in Web-based applications. As a result, evaluation of Web-based applications remains confounded by users' Web interaction challenges; their utility for specific user types remains unclear. Without appropriate evaluation of users' problems and challenges in using Web-based applications, we cannot begin to solve these problems and challenges. This paper demonstrates how TUME can be used to identify the unique problems and challenges of specific user types in using Web-based applications and suggests potential solutions. The outcome is an accurate understanding of specific design elements that present roadblocks and challenges for the user in interacting with the Web-based application and feasible design modifications to potentially improve the utility of these applications for specific user types.

We illustrate the application of TUME to test the utility of LMS for BVI students. We use a

Material published as part of this publication, either on-line or in print, is copyrighted by the Informing Science Institute. Permission to make digital or paper copy of part or all of these works for personal or classroom use is granted without fee provided that the copies are not made or distributed for profit or commercial advantage AND that copies 1) bear this notice in full and 2) give the full citation on the first page. It is permissible to abstract these works so long as credit is given. To copy in all other cases or to republish or to post on a server or to redistribute to lists requires specific permission and payment of a fee. Contact Publisher@InformingScience.org to request redistribution permission. popular LMS and an on-line exam as a representative task. We present results from three assessments that are essential components of TUME. Assessment I uses verbal protocol analysis to identify where and why BVI participants face LMS interaction problems in completing an exam. Assessment II performs text analysis of accessibility and usability design standards to identify design errors in the LMS exam environment. As- 
sessment III employs recursive abstraction analysis of Web developers' comments to understand why these problems occur and how they may be addressed feasibly. Together, these assessments show how TUME can identify design errors and their consequent problems for BVI users, the source and nature of these problems, as well as potential remedies for these solutions.

TUME provides researchers in education, human-computer interaction, and related fields a novel technique to understand the unique accessibility and usability needs of BVI users. Educators and education technologists may use this technique to accurately evaluate LMS for BVI students. With further validation and generalization across more problems, findings can be used to develop design principles for accessible, usable, and useful LMS for BVI students. Such design principles provide the basis to improve the efficacy of existing accessibility and usability standards for Web applications. TUME leads to improved accessibility and usability evaluation for non-visual interaction needed in mobile and ubiquitous computing environments and other multi-tasking situations.

Keywords: Learning Management Systems, Accessibility, Usability, Blind and Visually Impaired Students, Multi-Method Evaluation

\section{Introduction}

Learning Management Systems (LMS) are integral to today's education system (De Smet, Bourgonjon, De Wever, Schellens, \& Valcke, 2012). The adoption of LMS by academic institutions has grown significantly over the last ten years (Murray, Perez, Geist, \& Hedrick, 2012; Pynoo, Devolder, Tondeur, van Braak, Duyck, \& Duyck, 2011). More than $90 \%$ of American universities and colleges deliver academic programs using LMS (Arroway, Davenport, $\mathrm{Xu}$, \& Updegrove, 2010). A Sloan Consortium report "Going the Distance: Online Education in the United States, 2011" (http://sloanconsortium.org/publications/survey/going_distance_2011) found that over 6.1 million American students took an online course in Fall 2010. This represents a ten percent growth rate in 2010, which is the second lowest since 2002. The prevalence and wide adoption of LMS has appropriately motivated much research on its utility for students (Hamuy \& Galaz, 2010; Lonn \& Teasley, 2009; Van Raaij \& Schepers, 2008). However, very scant research examines whether blind and visually impaired (BVI) students can effectively use a LMS to derive desired learning outcomes (Babu, 2011). LMS often lack the accessibility and usability needed for non-visual interaction (NVI) (Armstrong, 2009), due to which completing e-learning tasks becomes difficult or impossible for BVI students (Babu, Singh \& Ganesh, 2010). This leads to an un-empowered BVI user who is discouraged and disengaged from the process - they just give up. While accessibility and usability have both technology and cognitive components (Babu, 2011), extant approaches are predominantly techno-centric. They directly translate Web content and controls into an audible format without paying adequate attention to critical elements of user cognition in Web interaction tasks. There is a critical need to holistically evaluate LMS accessibility and usability and identify feasible design solutions to improve LMS utility for BVI students.

The purpose of this paper is to present a novel task-oriented, user-centered, multi-method evaluation (TUME) technique and demonstrate how it can be used to evaluate and improve the accessibility, usability, and utility of LMS for BVI students. Task-orientation is practical as it links LMS accessibility and usability to its utility as an e-learning tool. User-centeredness explains how accessibility and usability problems create challenges in completing an e-learning task for users. The multi-method evaluation obtains and synthesizes multiple perspectives from multiple disciplines to provide a complete evaluation of problems in LMS accessibility and usability and suggest solutions to alleviate these problems. Specifically, the TUME approach emphasizes detailed analyses of BVI students' LMS interaction problems in completing an e-learning task, standardsbased assessment of the e-learning task environment, and designer analyses of problems and potential solutions. 
TUME is an innovative, multi-faceted approach to evaluating LMS. First, it purposefully integrates multiple methods and research from multiple disciplines for a more complete, practical, and solution-oriented evaluation of accessibility and usability. Traditional techno-centric approaches, including automated testing and expert technical review, assess the compliance of interface elements with de-facto design standards such as Web Content Accessibility Guidelines (WCAG) and Web Usability Heuristics. They do not adequately consider critical elements of user cognition as users engage in tasks that involve Web interaction. User-centric approaches (e.g., Babu et al., 2010; Leuthold, Bargas-Avila, \& Opwis, 2008; Theofanos \& Redish, 2003) target effective usability without adequate attention to technical sources of problems in Web interaction. TUME considers both the technical and the user-centric cognitive perspectives to provide actionable design guidance to improve LMS utility. Second, it represents a paradigm shift towards a cognitive view of accessibility and usability evaluation. Existing approaches focus on the perceivability of web interface elements and not their perceived affordance - what the user believes they can do with them (Babu, 2011). Specifically, extant approaches assume that Web sites become accessible and usable when they comply with standards such as WCAG and are interoperable with screen-reader software. TUME challenges this assumption by emphasizing the need to consider users' perceived affordance of Web interface elements for effective task completion. Third, it employs verbal protocol analysis to obtain users' revealed cognition, perceptions, and preferences while engaged in Web interaction tasks. Existing approaches are designed to obtain users' stated preferences and perceptions that do not provide a complete view of Web interaction problems. TUME is equipped for a more complete assessment of accessibility and usability. Fourth, it vets the design knowledge obtained from user-centered assessment from the perspective of a Web developer. Existing design standards such as WCAG are sometimes perceived as complex, ambiguous, and technically infeasible (Clark, 2006). Consequently, developers and designers fail to implement these effectively. TUME generates technically feasible design solutions based on Web developer and designers' perspectives.

The focus of this paper is to present and illustrate the application of the TUME technique to test the online exam environment of a popular LMS. We present the three qualitative assessment studies that are essential parts of TUME evaluation. Assessment I employs verbal protocol analysis to identify where and how BVI participants face LMS interaction problems in completing the exam. Assessment II performs text analysis of Web Content Accessibility Guidelines (WCAG) and Web usability heuristics to identify design errors in the LMS exam environment. Assessment III employs recursive abstraction of Web developers' analyses to develop an understanding of why these problems occur and how they may be addressed feasibly. Results demonstrate the efficacy and feasibility of the TUME technique in providing more complete, practically-relevant, and solution-oriented assessment of LMS accessibility and usability for BVI e-learners. The outcome is a clear understanding of specific design elements that present roadblocks and challenges in completing a LMS exam, and feasible design modifications to potentially improve LMS utility for BVI students.

While it is critical that Web sites and Web applications are designed for accessibility and usability, this must be achieved without undermining Web experiences and reducing Web functionality for any user group. This requires a Pareto-efficient approach that benefits one group without hurting others. For example, consider BVI users' inability to perceive graphical information. Eliminating all graphics from a Web page is impractical, as this could compromise usability for typical sighted users. However, effectively communicating information embedded in graphics through alternative non-visual formats is beneficial to BVI users without compromising any functionality. Effective communication means this alternative format enables the BVI users to perceive, understand, and use the information to achieve their goals. This multimodality has beneficial implications for the elderly, the dyslexic and users with other disabilities. In fact, research demonstrates that technology designed for BVI accessibility and usability is more usable for typical sighted 
users (Jana, 2009). Innovative organizations make strategic investments to improve the usability of their products by partnering with the BVI as power users. Apple's VoiceOver technology and NaturallySpeaking are prime examples of such well positioned strategic investments to develop accessibility and usability solutions for all. TUME specifically aims to improve Web accessibility and usability for BVI users, and at the same time improve the utility for all.

TUME provides researchers in education, human computer interaction, and related fields a novel technique to understand the unique accessibility and usability needs of BVI users. Educators and learning technologists may use TUME to accurately evaluate their e-learning LMS implementations for BVI students. With further validation and generalization across more problems, the evaluations presented here can provide the basis to develop design principles to guide the development of accessible, usable, and useful LMS for BVI students. In addition, these design principles can provide the basis to improve the efficacy of existing standards on Web accessibility and usability (e.g., WCAG) for the BVI users. It will lead to improved accessibility and usability evaluation for non-visual interaction (NVI), which is used by all users in mobile and ubiquitous environments (Brown \& Kaaresoja, 2006; Lumsden \& Brewster, 2003), as well as in multitasking situations (Christian, Kules, Shneiderman, \&Youssef, 2000).

The remainder of the paper is organized as follows. We review existing literature to better understand current evaluation approaches and motivate the need to search for a new approach. We then explain the merits of our task-oriented, user-centered, multi-method evaluation approach. We next discuss our research design to test the efficacy and feasibility of this approach. Following this, we present our analysis. We then discuss the practical implications of our findings. We close with our conclusions and discuss directions of on-going and future research in this area.

\section{Literature Review}

Here, we briefly review the literature on the evaluation of accessibility and usability and its implications on the utility of Web-based applications for users, to help orient the reader. We describe accessibility and usability and provide a brief review of the literature on traditional accessibility and usability evaluation of Web-based applications.

\section{Accessibility and Usability}

Accessibility and usability are two related but distinct concepts. Accessibility allows users access to system functionality (Goodhue, 1986). For users with disabilities, accessibility is treated as a technical construct that allows assistive technologies, such as screen-readers, the necessary access to interface elements (Leuthold et al., 2008). Usability refers to how well a system conforms to users' conceptualization of performing a task using it (Goodwin, 1987). It is a cognitive construct that depends on the task the user performs. A system that is not accessible is not usable; however, an accessible system does not guarantee usability (Di Blas, Paolini, \& Speroni, 2004). Accessibility problems prevent access to system features and functionality. Usability problems prevent the use of these features and functionality to meet objectives. Therefore, systems accessibility and usability are key to deriving the utility of a system.

\section{Traditional Accessibility and Usability Evaluation}

Traditionally, the accessibility and usability of Web-based applications, including LMS, are evaluated using the Web Content Accessibility Guidelines (WCAG). WCAG is the de facto standard on Web accessibility and usability for users with disabilities (Kelly, Sloan, Phipps, Petrie, \& Hamilton, 2005). It comprises a set of design guidelines and success criteria principles established by the World Wide Web Consortium (W3C) Web Accessibility Initiative (WAI). WCAG guidelines-- perceivability, operability, understandability, and robustness-represent four Web 
accessibility principles. WCAG success criteria are considered normative and include definitions, benefits, and examples. WCAG forms the basis for regulations and laws on equal access such as Section 508 of the U.S. Rehabilitation Act.

WCAG guidelines and success criteria also inform traditional standards-based evaluation methods such as automated testing, expert technical review, and user testing. Automated testing uses accessibility checkers to identify interface elements that violate WCAG requirements. Better tools provide explanations of why the individual elements violate WCAG. Expert technical review uses experts to examine representative Web pages for WCAG compliance (Mankoff, Fait, \& Tran, 2005). User testing asks representative users to perform tasks and respond to questions on accessibility and usability as defined by WCAG (Correani, Leporini, \& Paternò, 2004).

\section{Learning Management Systems}

Learning management systems (LMS) are also referred to as Virtual Learning Environments, Digital Learning Environments, Course Management Systems, and Electronic Learning Environments in the literature (De Smet et al., 2012). An LMS is a web-based application designed to support training, teaching, and learning on-line (McCormick, 2000). It allows students to accomplish course activities such as reading course material, completing assignments, taking exams, conducting Internet research, participating in class discussions, and working on group projects on-line (Pituch \& Lee, 2006; Sun, Tsai, Finger, Chen, \& Yeh, 2008). LMSs have become an integral part of today's academe (Arroway et al., 2010).

\section{Blind and Visually Impaired Students}

BVI students lack the sight necessary to see information presented on a computer screen. They cannot operate a mouse. They rely predominantly on text-to-speech software called screenreaders (SR) to interact with computers and the Internet (Lazar, Allen, Kleinman, \& Malarkey, 2007). They use key commands (including keyboard shortcuts afforded by the operating system and specialized keystrokes afforded by the screen-reading technology) to operate the SR. The SR identifies and interprets text content on the computer screen and presents this aurally through a synthetic voice (Di Blas et al., 2004). Jaws, WindowEyes, VoiceOver and NVDA are commonly used SRs. JAWS (www.freedomscientific.com) is a market-leading, comprehensive SR for BVI individuals including users with blindness, low vision or other vision-related impairments.

BVI students represent an atypical population with special functional and learning needs. In this paper, we apply TUME to focus on the special e-learning needs of this atypical student population. An analysis of the literature indicates that Web interaction for BVI students has the following characteristics:

i. Information access is sequential (Lazar et al., 2007). At any given point, BVI users perceive only a snippet of the content and often lose contextual information.

ii. No rendition of graphics (Armstrong, 2009). BVI users cannot perceive or interpret information communicated through images, color, and layout.

iii. Quick information scan is not possible (Di Blas et al., 2004). BVI users cannot locate goal-relevant information efficiently and easily by scanning information.

iv. Keyboard-based (Leuthold et al., 2008). BVI users cannot use functionality that requires mouse input.

v. Complex layouts create problems. When Web pages have a complex layout, screenreader's feedback becomes ambiguous (Lazar et al., 2007). Screen-readers also mispronounce many words (Theofanos \& Redish, 2003) which create comprehension problems for the BVI user.

vi. Requires learning complex interface. BVI interaction requires memorizing hundreds of key commands (Theofanos \& Redish, 2003). The wide range of screen-reader functional- 
ity makes it more difficult for BVI users to remember and use appropriate functions for effective Web interaction.

vii. Higher cognitive load. Cognitive resources must be split, trying to understand the browser, the screen reader, and content (Theofanos \& Redish, 2003). This leads to greater cognitive burden for BVI users in Web interaction (Millar, 1994; Thinus-Blanc \& Gaunet, 1997).

Students accomplish learning activities using LMSs and other Web-based tools such as elibraries, and online encyclopedias (Alavi \& Gallupe, 2003). Research shows that these Webbased tools lack the accessibility and usability needed (American Foundation for the Blind, 2008; Babu \& Singh, 2009; Brophy \& Craven, 2007). The problem is especially debilitating for BVI users (Leuthold et al., 2008). Poorly designed learning environments negatively impact their academic outcomes. They require learning environments that are accessible, usable, and useful for elearning.

While LMS are designed for typical sighted students, it is believed that BVI students too can maximize their educational outcomes through their effective use (Kim-Rupnow \& Burgstahler, 2004). For all students, and particularly the BVI student, effective LMS use requires accessibility and usability (Babu \& Singh, 2009). In fact, BVI students perceive LMS accessibility and usability as key to academic success in online education, yet this is often lacking in available LMSs (Babu, Singh, Iyer, \& Midha, 2007). Our research tries to understand LMS accessibility and usability for BVI students so that they can maximize the educational benefits and academic outcomes from LMS use.

Existing standards-based evaluation approaches are necessary but not sufficient for an accurate assessment of Web accessibility and usability (Clark, 2006; Di Blas et al., 2004; Mankoff et al., 2005). They identify interface elements that prevent access to screen-readers and exhibit poor usability (Kelly et al., 2005; Moss, 2006). However, they are not able to explain the consequent problems for the BVI user in goal accomplishment. Experts believe WCAG does not accurately represent usability needs of the BVI user (Clark, 2006; Di Blas et al., 2004; Leuthold et al., 2008). For instance, the perceivability guideline recommends that the graphical interface should be modified to facilitate screen reader access (Leuthold et al., 2008). However, the effectiveness of the common implementation of this guideline is questionable. Content readability for the BVI is typically implemented through aural presentation of content that provides partial assessment of LMS accessibility or usability (Di Blas et al., 2004). A WCAG-based evaluation alone provides an incomplete account of accessibility and usability problems in Web design for the BVI user.

Prior research (Babu et al., 2010) demonstrates a novel, user-centered, and cognitive method to evaluate Web accessibility and usability using verbal protocol analysis (VPA) for assessment of Web-based application from the BVI users' perspective, including where and how problems arise in completing an online tasks. Although this user-centric method develops information not available through the techno-centric standards-based evaluation, it does not identify the source of the problem. Nor does it provide actionable guidance on potential solutions.

Evaluation techniques that do not provide feasible and actionable guidance on how to address users' accessibility and usability problems have limited utility for generating design improvements. Evaluation methods that incorporate the perspectives of Web developers and designers can identify problems as well as solutions to potentially alleviate specific problems. Developers and designers have a more accurate understanding of the systems model (Norman, 1988). They can help trace the source of a BVI user's Web interaction problem to a component of interface design. They can provide valuable insights leading to actionable guidance on improving accessibility and usability. Scalability of such problem-specific solutions is a challenge. A practically-relevant, 
solution-oriented evaluation of Web accessibility and usability must consider perspectives of Web developers/ designers, BVI users, and WCAG.

A holistic LMS evaluation is critically needed to accurately understand accessibility and usability problems BVI students face in e-learning. It is important to note that while we study BVI students' accessibility and usability problems, the consequent accessibility and usability improvements should be useful for all, including the sighted. Our research studies LMS accessibility and usability in the context of its utility as a learning platform. The research question addressed in this paper is: How can we effectively synthesize a user-oriented perspective with existing standards such as WCAG as well as the Web developers and designers' assessment for a complete, practically-relevant, solution-oriented evaluation of accessibility, usability and derivable utility of Learning Management Systems for Blind and Visually Impaired students?

\section{The TUME Approach}

This paper presents a novel task-oriented, user-centered, multi-method evaluation (TUME) approach for a holistic, practical, solution-oriented assessment of accessibility, usability, and utility of Web-based applications.

\section{Task-Oriented}

The task-orientation helps examine the accessibility and usability of a Web-based application in terms of its utility. Web-based applications are designed to serve a purpose. For example, an LMS is designed to support e-learning tasks. On the other hand, Web interaction is goal-oriented; users visit a Web site to accomplish a task. For example, students interact with an LMS to complete their homework. A measure of the success of the Web-based application is how effectively users can complete the tasks it is designed to support. When accessibility and usability problems hamper task accomplishment, the Web-based application fails to achieve its purpose. A more practical approach to accessibility and usability evaluation should contextually situate these problems in the purpose of the Web-based application. This will allow the examination and explanation of design errors beyond the identification of poorly designed interface elements, to their implications for users' ability to complete a task supported by the Web-based application. Contextually situating the evaluation in the task brings together accessibility and usability of a Web-based application to its utility. This has greater practical relevance in terms of the ability to develop solutions to enhance the utility of the application. Our approach provides contextually situated evaluations where a Web-based task is the unit of analysis, as opposed to interface elements on a web page. This allows us to identify accessibility and usability problems in Web-based applications as well as the consequent challenges in completing its supported tasks. This provides a more complete and contextually-situated understanding of the problem.

\section{User-Centered}

Extant research on Web accessibility and usability for users with different abilities (e.g., the BVI, the elderly, etc.) is predominantly techno-centric in nature. It focuses on assistive-technologywebsite interaction, as opposed to user-website interaction (Babu, 2011). It assumes that people with different abilities are typical users in every other way, except they interact with Web-based applications using specific assistive technologies (Correani et al., 2004). Thus, in this approach, it follows that accessibility and usability are served when Web content interoperates with the myriad assistive technologies. It attributes Web interaction problems to interface elements that are inaccessible to the user's assistive technology (Freire, Goularte, \& Fortes, 2007). Technical approaches may identify accessibility issues that are only part of the problem. They address this problem through improvements in interface design and assistive technology. They require users with different abilities to adapt their interaction strategies to the modified design (King, Thatcher, 
\& Easton, 2005). In contrast, user-centered approaches focus on generating new design knowledge and design improvements from careful examination of users' interaction challenges. They consider cognitive factors such as problem-solving, perception, memory, and learning in systems interaction (Katz-Haas, 1998). They place the user's needs, preferences, and abilities at the core of interface design (Greenbaum \& Kyng, 1991; Schuler \& Namioka, 1993). This yields a more accurate assessment of systems usability in terms of user needs (Heim, 2007; Krug, 2005). We adopt a user-driven approach to study users' challenges and roadblocks in Web interaction resulting from accessibility and usability problems in the design of a Web-based application.

\section{Multi-Method Conceptualization of Accessibility and Usability Evaluation}

Our multi-method approach performs a more complete evaluation of accessibility and usability by combining the perspectives of users, designers, and prevalent design standards. At the core of this multi-method approach is a tripartite involvement of three entities: the user, the WCAG standards, and the Web developer/designer. The user is either typical or atypical. An atypical user could be BVI, elderly, dyslexic, hearing impaired, or have any other different abilities. Web experience for each user group is unique. For example, Web interaction is a listening activity for the BVI user, instead of a visual activity. Each user group has a unique set of accessibility and usability needs in Web interaction. WCAG is the de facto standard on Web accessibility and usability. It governs how a Web-based application accommodates the unique Web interaction needs of specific user groups. Developers incorporate WCAG recommendations into their Web sites for accessibility and usability. Figure 1 shows our conceptualization of Web accessibility and usability evaluation using BVI as an example.

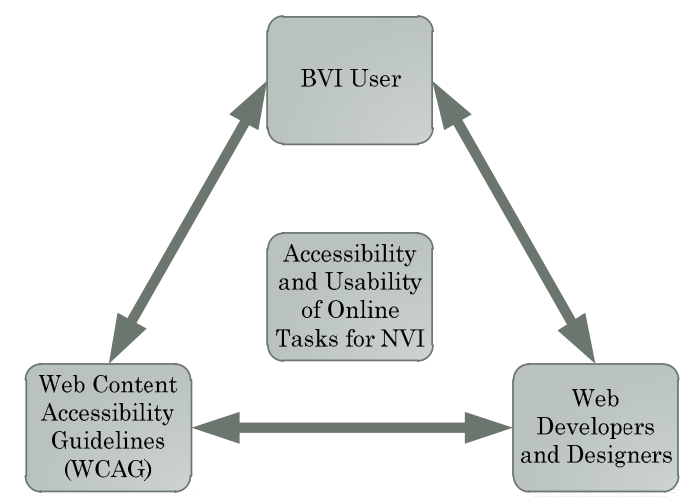

Figure 1: Multi-method conceptualization of Web accessibility and usability for BVI.

Web accessibility and usability is a cognitive construct that emerges from the shared understanding of special needs. Accessibility and usability problems occur due to discrepancies in the user's understanding of how a Web-based application works and how this Web-based application actually behaves. In other words, Web interaction problems arise due to discrepancy in the users' expected and observed outcomes of actions in completing a Web-based task (Babu et al., 2010). Discrepancies in the expected and observed outcomes of user action on a Web-based application can be understood in terms of Donald Norman's (2002) two kinds of gulfs:

Gulf of execution: represents the discrepancy between a user's intentions and the system's allowable actions. Consequently users face difficulty translating goals into actions. This is typically manifest in accessibility problems. 
Gulf of evaluation: represents the discrepancy between a system state and the user's ability to perceive and understand that state directly with respect to expectations. It is large if feedback is difficult to perceive, difficult to understand, and is inconsistent with user's expectation. This is typically manifested in usability problems.

We conceptualize Web accessibility and usability problems as users' difficulties due to discrepancies between the expected and observed outcomes of their actions in completing a Web-based task. Using Norman (2002) as a theoretical foundation, we refer to difficulties resulting from gulf of evaluation as dissonance, and those resulting from gulf of execution as failure. Analyzing dissonance and failure from the perspective of accessibility and usability principles (e.g., WCAG) helps to understand their nature. Analysis from the perspective of developers and designers helps identify design problems and understand their nature. Together, these provide a more complete and utilitarian view of accessibility and usability of a Web-based application. We argue that our task-oriented, user-centered, multi-method approach is needed for an accurate, practicallyrelevant and solution-oriented evaluation of Web-based applications for specific user types not possible through current approaches. We present our method and research design to demonstrate the application of our novel evaluation approach.

\section{Methodology and Research Design}

We applied our TUME approach to evaluate the accessibility, usability, and utility of a learning management system (LMS) for blind and visually impaired (BVI) students. LMSs allow students to complete e-learning tasks. BVI students comprise an atypical user population that interacts with LMSs by listening to its content read aloud by screen-reader assistive technology. We employed TUME to test LMS accessibility and usability in terms of its utility as an effective learning tool for this atypical user population. We performed a contextually situated evaluation where an e-learning task was the unit of analysis, instead of interface elements on an LMS page. This helped us identify accessibility and usability problems in LMS design, the consequent challenges for BVI students in completing e-learning tasks, as well as a more complete and contextuallysituated understanding of the problem. Such understanding will guide future research to improve the functional and academic outcomes for BVI students in online education.

We used an online exam as a typical and common context of LMS use. The unit of analysis was an online task, which included a multiple-choice question, a multiple-answer question, and an essay-type question. It should be noted that these represent the three most common formats of presenting questions in online exams (Simon \& Cutts, 2012), as well as in Web-based surveys, online job applications, and online college applications (Neumeier, 2005). In addition, they represent the standard forms of information input for web forms. Therefore, while the context of the study was a particular LMS, the accessibility and usability problems identified here are conceivably common to other forms of entering information on the web.

The online exam included 12 distinct activities spread over 6 pages of the LMS. Figure 2 shows the prescribed set of activities that comprise the workflow to complete the exam. 


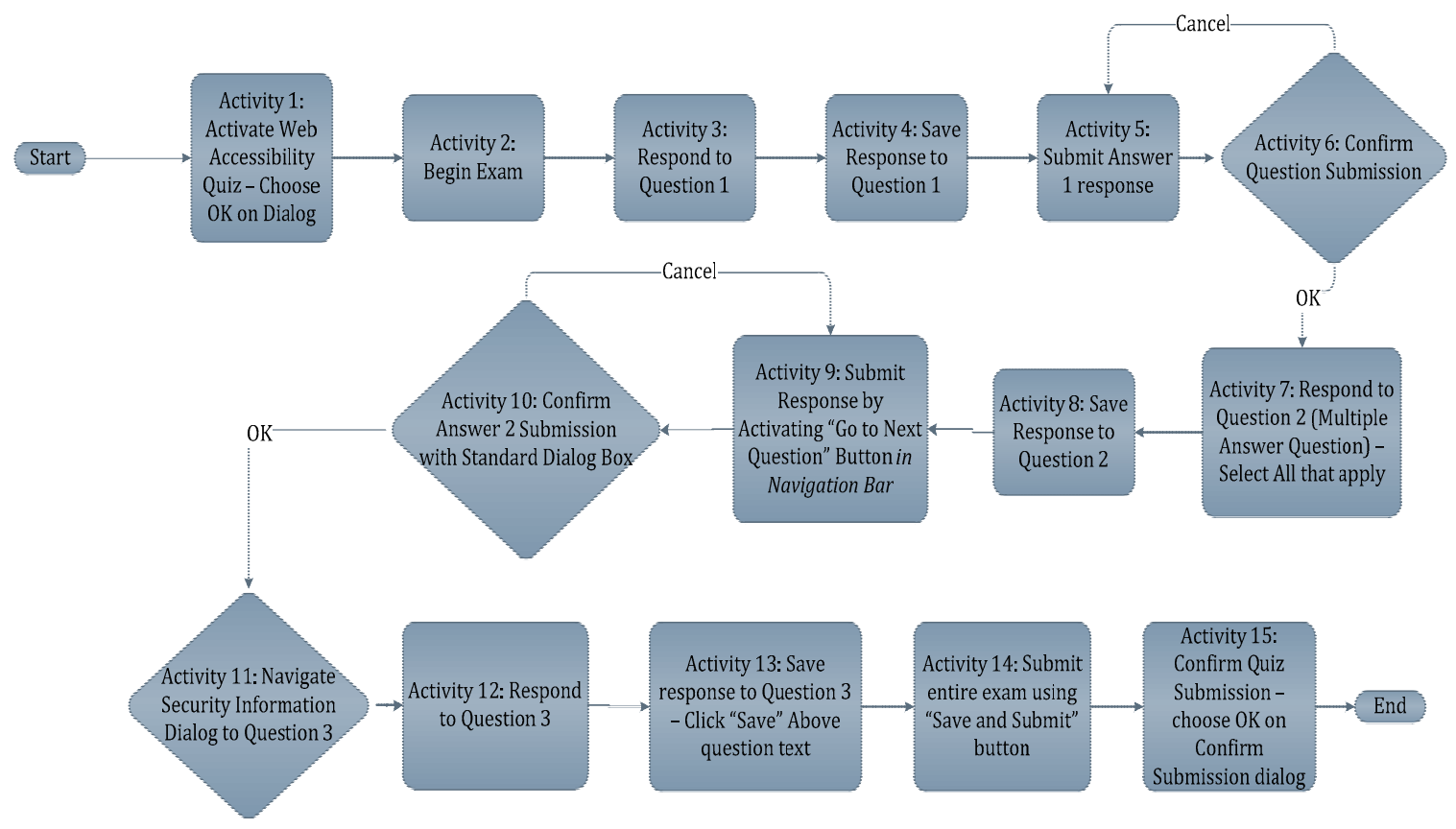

Figure 2: LMS Activities used for assessment.

Activity 1. Activate the link "Web Accessibility Quiz" to bring up the exam. The Web Accessibility Quiz link opened a page with the message "Click OK to begin the quiz," and a button labeled "OK" as the only command choice on the page.

Activity 2. Begin the exam by choosing OK, which opened a new page with a multiplechoice question. The page presented a set of instructions, possible number of points, a link named "Save", Question 1 text, four radio button options and the navigation bar.

Activity 3. Respond to Question 1 by selecting a radio button corresponding to one of the four options.

Activity 4. Submit response by activating the "Go to Next Question" button in the navigation bar. A "Confirm Question Submission" box opened which is a standard dia$\log$ box with an "OK" and a "CANCEL" button.

Activity 5. Confirm Answer 1 submission by activating the "OK" button. Selecting OK brought up a new page that displayed instructions, possible number of points, a link named "Save", Question 2 text with four options, and the navigation bar. Question 2 was in the multiple-answer format.

Activity 6. Respond to Question 2 by selecting checkboxes corresponding to all options that apply.

Activity 7. Submit the response by activating the "Go to Next Question button" in the navigation bar.

Activity 8. Confirm Answer 2 submission by activating "OK" button in a "Confirm Question Submission" dialogue pop-up - a standard dialog box with "OK" and "Can- 
cel" options. Choosing "OK" brought up a "Security Information" dialogue box. It contained a security warning message, a "Yes" button and a "No" button.

Activity 9. Get past the Security warning by choosing "Yes" and move to a new page. The new page includes five relevant components: (1) instructions on completing the exam; (2) possible number of points; (3) link "Save"; (4) question 3 text; (5) several text formatting controls; (6) input area; (7) additional controls leading to the navigation bar; (8) navigation bar with a "Save and Submit" button instead of the usual "Go to Next Question" button

Activity 10. Respond to Question 3 by locating the input area and typing in the answer.

Activity 11. Submit the entire exam by activating the "Save and Submit" button.

Activity 12. Confirm exam submission by choosing "OK" on the "Confirm Submission" dialogue box. The last page loads that displays a message confirming submission and grade information.

The TUME application described in the following sections includes:

Method I: BVI student assessment using verbal protocol analysis;

Method II: WCAG assessment using textual analysis;

Method III: Web developer assessment using structured, open-ended interview.

\section{Method I: BVI Student Assessment Using Verbal Protocol Analysis}

We used the think-aloud method of direct observation, also called concurrent verbal protocol analysis, to collect concurrent verbal reports of BVI students. Participants work on a task and concurrently verbalize whatever they are thinking (Ericsson \& Simon, 1984; Todd \& Benbasat, 1987). Concurrent verbal reports contain evidence of the information that participants process to perform a task (Ericsson \& Simon, 1984). Ericsson and Simon (1993) found that concurrent verbalizations are non-reactive and do not alter participants' behavior in tasks. This technique is effective for developing an in-depth understanding of human problem-solving (Newell \& Simon, 1972) and is a feasible method to trace usability problems in e-learning tools (Cotton \& Gresty, 2006).

\section{Participants}

We recruited six BVI participants (Mean Age $=23$ years) including five males and one female. While participants had typically used the Web for email and information gathering for over 5 years, none had used a LMS or taken an exam online. Three participants were high school seniors at the Texas School for the Blind and Visually Impaired (TSBVI), two high school seniors and a college freshman were affiliated with the Michigan Commission for the Blind Training Center (MCB/TC) at the time of the study. Technology instructors at both institutions, who train students in using screen-readers, computers and the Web, facilitated participant recruitment.

\section{Material}

Materials for Method I assessment included instructions, a course designed for this study, and an online exam in the LMS. Participants log on to the LMS and visit the course. The "Announcements" section in the course directs participants to the Assignments section, which provides instructions on completing the online exam called "Web Accessibility Quiz." 


\section{Procedure}

We conducted a familiarization session with participants to describe the study's objective and explain the think-aloud technique and answered questions to ensure they understood the methodology. Participants then took the LMS exam, which lasted for approximately 45 minutes. We recorded and transcribed verbal protocols including participant verbalizations, conversation, and screen-reader audio.

We performed verbal protocol analysis on the transcripts and decomposed them into individual segments that represent single units of thought. We identified segments that represented a Web interaction challenge that hampered a participant's ability to perform an activity effectively and labeled them Problems. Problems where the situation was not comprehensible to the participant due to inadequate system feedback were labeled Dissonance. We identified problems corresponding to a situation where things did not work for the participant - an action did not yield expected outcome. We labeled this category of problems as Failure.

\section{Method II: WCAG Assessment Using Text Analysis}

Method II identified accessibility and usability problems using WCAG Success Criteria and Usability heuristics respectively. The objective of Method II was to interpret findings of Method I into design guidance.

\section{Material}

Materials included Method I results, WCAG guidelines and success criteria, and usability principles/heuristics applicable for BVI students. Specific web guidelines employed are Perceivability, Operability, Understandability, and Robustness. We also adopted usability principles/heuristics for BVI users (Nielsen, 1993); Principles of Good Design (Norman, 2002); and Golden Rules of Interface Design (Shneiderman \& Plaisant, 2004).

\section{Procedure}

We used WCAG guidelines and related usability criteria to determine the accessibility and usability character of a problem. We analyzed individual WCAG success criterion to understand problems by retracing BVI participants' LMS interaction paths and identified design elements used for the task. This provided a mapping between problematic LMS design elements and the accessibility criteria violated. We analyzed usability principles to explain problems, which provided a mapping between problematic LMS design elements and the usability criteria violated. Together, these helped explain BVI students' accessibility and usability problems.

\section{Method III: Web Developers Assessment of Online Exam Using Structured, Open-Ended Interview}

\section{Participants}

We recruited five Web developers, having a minimum of three years Web development experience.

\section{Material}

Materials included results from Method I assessment, task environment of the LMS exam, and an interview protocol informed by the results of Method I. Interview questions include:

1. Based on your understanding of the roadblock/challenge experienced by BVI participants, what do you think is the problem source? 
2. What are some possible solutions (e.g., design modifications) you believe can be feasibly implemented?

\section{Procedure}

We conducted one-on-one interviews with Web developers where we explained the purpose of the research and presented the task environment and briefed them about BVI assessment and described each activity where participants faced a roadblock or a challenge. For consistency and accuracy, we described each scenario and asked participants to retrace the complete path of BVI participants, examine the task environment corresponding to each activity, explain the cause of the problem, and suggest possible solutions.

We analyzed the interview data using recursive abstraction. In recursive abstraction, qualitative evidence is distilled to obtain knowledge through a process of summarization without using codes (Crabtree \& Miller, 1999). We divided each transcript into segments based on developers' understanding of the problems and their suggested solutions. We summarized and re-summarized information directly relevant to the questions for each participant. These summaries included statements that attribute a problem experienced by BVI participants to interface elements of the online exam and statements that identify remedial measures to address problems in terms of feasible design modifications.

Synthesizing the results of the three assessments provides our approach the ability to generate a more holistic understanding of LMS accessibility and usability problems in terms of BVI students' ability to complete online exams. We hope that the approach is extensible to other user groups of different abilities and other modes of LMS interaction for typical and atypical user groups.

\section{Results and Discussion}

Developing a better understanding of BVI students' unique accessibility and usability problems was the desired outcome of our TUME LMS evaluation. The BVI student assessment provided an in-depth, observational and experiential understanding of problems. The WCAG assessment explained the accessibility and usability character of these problems. The Web developer assessment further clarified the nature of the design problem, and identified potential design solutions.

We grouped the results of our TUME LMS evaluation into multiple problem types. In the following, we discuss three primary problem types in LMS design: Inconsistent feedback; inaccessibility of security pop-ups; and obscurity of input areas for answer entry. We explain these problem types in terms of specific assessments.

\section{Inconsistent LMS Feedback for Link Activation}

The first significant problem in LMS design for screen-reader users is inconsistent system feedback for link activation. We explain the nature of this problem using results from the three assessments

\section{Results of BVI student assessment of LMS design}

A significant challenge for BVI students is confusion when navigating across different LMS pages. Very often, participants were not aware that their destination page had become available after they clicked on the related link on the source page. Therefore, they either kept waiting to interact with the new page or would interact assuming it was the previous page. This confusion, coupled with the resultant frustration, was frequently evident. We provide evidence of such confusion using verbal reports collected from two participants. Participant's verbalizations are la- 
beled BVI \#, screen-reader output as SR, and questions to participants as Q. We wish to remind the reader that BVI users operate the SR using key commands that include generic keyboard shortcuts afforded by the operating system and specialized keystrokes afforded by the screenreading technology. The SR responds verbally with typing echo as well as with announcement of the text content at cursor focus.

SR: $\quad$ Link Web accessibility quiz

BVI2: $\quad$ Ok, web accessibility quiz. I am going to hit enter on this.

SR: $\quad$ Enter. Web accessibility quiz visited link. Frame.

BVI2: $\quad$ Once again, I entered into the same problem. It didn't tell me I have entered into a new page. It didn't say page has how many links. It just said frame. I don't know what that means. But I clicked on a link. And I assume it worked.

These show the confusion and uncertainty in the mind of the BVI user due to difficulty in knowing if link activation was successful and if a new page loaded. This confusion seems to stem from a discrepancy between observed and expected feedback for link activation. Actually, the expected feedback is indicative of a "cultural convention" in Web navigation for screen-reader users. According to this convention, after successful link activation, a screen reader typically announces the percentage of the destination page content downloaded when a link is activated; and the page composition, such as "Page has x links, y images, $\mathrm{z}$ tables," referring to specific interface objects in the destination page. A BVI user interprets the percentage value announcement as the progress of page download. Since BVI students cannot perceive visual cues, these announcements are critical to detect a change of system state, such as an LMS page change. If any part of this announcement is missing, the student remains in the "dark". We observed multiple occurrences of such interruptions when participants tried to navigate LMS pages. Another example is presented below:

BVI2: $\quad$ I am going to go back to the bottom of the page with control end, and scroll up to complete the quiz.

SR: $\quad$ Click ok to begin the quiz.

BVI2: $\quad$ Just click ok to begin the quiz. I am going to hit ok.

SR: $\quad$ Ok link graphic. Ok.

BVI2: $\quad$ Once again, I have no indication whatsoever from the speech program that I am starting the page, updating the page. So frustrating. I have made note of that several times, so I am not going to continue doing this in each page. But it is somewhat frustrating. When you do click on a link, it is not saying you have arrived on a new page. It just doesn't say anything at all.

In the above situation, the BVI student does not receive any feedback and cannot perceive the LMS's state. Consequently, he loses precious exam time in dealing with the uncertainty and confusion.

Another example of the confusion and uncertainty under such situations is:

SR: $\quad$ Blank link web accessibility quiz.

BVI4: $\quad$ Web accessibility quiz! I was looking for that. Enter on that.

SR: $\quad$ Enter. Content frame updated. Content frame end blank.

BVI4: $\quad$ What the heck! It's the contact, it's saying something about contact. I don't know what it's like. Maybe I should go up some?

SR: $\quad$ Link graphic cancel.

BVI2: Oops! 


\section{SR: $\quad$ Click okay to begin colon web accessibility quiz. \\ BVI4: Oh! \\ SR: $\quad$ Blank heading level}

In this case, the screen-reader announces the percentage consistent with the conventional announcement for link activation and announces "Content frame updated", consistent with the page composition announcement convention. The LMS generated feedback. However part of the feedback was inconsistent and this created confusion in the mind of the participant. The student could not interpret part of the announcement regarding the new page and could not verify if the new LMS page had loaded. She could not determine the next course of action and got frustrated.

LMS interaction problems are indicative of a gulf between the user's cognition of the LMS and its actual behavior. Confusion in navigation indicates a gulf between the expected and observed LMS behavior in link activation. BVI participants perceive the feedback as incomplete, ambiguous, and misleading. They spend extra time to verify their context and situation, which adversely impacts timely exam completion and their performance. According to the Action Model (Norman, 2002), this represents a gulf of evaluation as BVI students fail to interpret LMS feedback for their actions. Inconsistent feedback for link activation is a serious problem in LMS design that creates confusion and frustration for BVI students in navigation.

\section{Result of WCAG assessment of LMS design}

Inconsistent LMS feedback for link activation violates three design principles on Web accessibility and usability. These design principles include:

- $\quad$ Page Title - principle of Web accessibility;

- Feedback - principle of Web usability; and

- Satisfaction - principle of Web usability.

Violation of the Page Title principle: This principle says that Web pages should have descriptive titles for BVI users to orient themselves for this content (http://www.w3.org/TR/UNDERSTANDING-WCAG20/navigation-mechanisms-title.html). BVI users perceive information by listening to screen-reader announcements. They fail to identify the page or find goal-relevant information when the Web page lacks a descriptive title. This happened to our participants. They could not identify an exam page or understand its purpose from the screen-reader announcements. In our analysis, none of the exam pages had a descriptive title accessible to a screen-reader in violation of the Page Title principle for Web accessibility.

Violation of Feedback principle: This principle (Norman, 2002) highlights the need for "full and continuous" system feedback that informs users of the result of their actions on the system. Analysis shows the significant difficulties BVI participants faced in comprehending LMS feedback for link activation. Screen-reader announcements were incomplete in describing LMS state changes after interaction in violation of Norman's (2002) Feedback principle of Web usability.

Violation of Satisfaction principle: This principle (Nielsen, 1993) highlights the importance of user satisfaction with the system and its function. Our analysis demonstrated how BVI participants were frustrated with the LMS for not responding appropriately after activating a link. The feedback from the screen-reader was often perceived as confusing, incomplete, and misleading and created disruption. Inconsistent LMS feedback for link activation problems represents a violation of Nielsen's (1993) Satisfaction principle of Web usability.

Thus, inconsistent feedback for link activation is both an accessibility and usability problem in LMS design. 


\section{Results of Web developer assessment of LMS design}

Web developers' assessment explains that the source of the inconsistent feedback for the link activation problem is the frame-based page structure in LMS design. This frame-based structure failed to generate the conventional screen-reader announcement that follows link activation. Analysis shows these frames were not assigned unique titles. Screen-reader announcements failed to communicate a context change effectively. These contribute to BVI students' confusion. Web developers explained:

"Traditionally, a Web page update occurs through HTML. The Web site consists of content wrapped around something called an HTML body. Browsing to a new page means new content is loaded into the HTML body. In such a scenario, the screen reader announces $1 \%, 10 \%, 50 \%$ etc. However, in this particular case, the LMS loads new content through another means called frames. Frames basically divide the whole body into multiple parts, such as body 1, body 2, body 3, body 4, in that way. And what they do is, they only update body 3 which is relevant to you, and do not update body 1, body 2 and body 4. So, only part of the body is updated. Essentially, over here it means that page has changed and yet the page has not changed. The screen reader may not be capable enough to announce the frame data changes."

Each LMS page consists of a set of frames, including a header frame and a content frame. The content frame in turn comprises a menu frame and a course content frame. Course Content frames are used for dynamic components of an online exam, such as questions, possible answers, input areas, and Menu frames are used for static content such as menu items. LMS pages change by loading new content in the course content frame without changing content of other frames. In this respect, frame-based sites are different in that a new page involves loading new content into the HTML body. This difference may not be apparent to users, but it does alter screen-reader responses because the screen-reader does not perceive a change in the page. It may detect a change in the content of one frame and may not have information to announce the arrival of a new page. The screen-reader may announce "Content Frame Updated" - such announcements break convention and may create confusion in the minds of BVI students while navigating exam pages.

Participants discussed feasible design modifications as potential solution for BVI students' confusion such as the use of Accessible Rich Internet Application (ARIA) tags to improve the accessibility of dynamic Web content and advanced user interface controls that use Ajax, HTML and JavaScript. ARIA tags add attributes to identify features for user interaction, how they relate to each other, and their current state. ARIA describes new navigation techniques to mark regions and common Web structures, such as menus, primary content, secondary content, banner information, and other types of Web structures. For example, with ARIA, developers can identify regions of pages, allowing screen-reader users to easily move among regions, rather than having to press Tab many times. Since the LMS relies on the use of dynamic frames for organizing exam questions, the suggestion of WD1 has value. He explained:

"It is definitely possible to indicate that there are changes happening using ARIAAccessible Rich Internet Applications. They have a whole bunch of tags, including tags specifically designed to inform the user about a change. It is possible to extend this to pages with frames, and provide some additional information about the new content."

Being more specific about such design modifications, participant WD3 explained:

"For example, the body is divided into 4 frames-three frames are not updated; only 1 frame is updated with its content. Now, the screen reader will say 1 frame has 
changed, and convey to the user "This area of the body has changed. "Developers could even include frame names like "Main Content", "Side Bar", "Top Bar", etc. Accordingly, the Screen Reader will read the frame name and tell the user exactly which one has changed."

Developers attribute the inconsistency in LMS feedback to frame-based structures in LMS design. Absence of descriptive titles in dynamic frames compounds the problem for BVI students. When they activate a link on the source LMS page, the only thing that changes is the course content frame. This occasionally prompts the screen-reader to announce "Frame Four, Course Content Updated". However, because this announcement was (a) unconventional, (b) occasional, and (c) devoid of descriptive frame titles, it failed to effectively communicate to BVI students the identity of a newly loaded exam page, hence the confusion. Web developers also inform that the confusion problem can be potentially addressed through simple modifications in LMS design including unique and descriptive labels in the $<<$ Title Attribute $>>$ of each content frame and the use of ARIA tags to prompt the screen-reader to announce the descriptive label of the new frame loaded. From Web developers, we gather that with these design modifications, online exams could become more accessible and usable for BVI students. Moreover, these are very feasible and actionable design modifications.

\section{Inaccessible Security Information Pop-Up}

A significant problem in LMS design for BVI users are inaccessible security dialogue boxes.

\section{Results of BVI student assessment of LMS design}

A significant challenge for BVI students is their inability to access the security information popup and complete the online exam. Participants were unable to perceive, understand, or operate the dialogue box. We observed their failure to get past the security information dialogue box. This dialogue box appears immediately after a page with essay-type question loads and restricts the access screen-reader to itself - the user cannot navigate out of the dialog box. The only information participants hear is a "Yes" or "No" button. They could not understand the purpose of the dialogue nor decide what to do. Participants got trapped in the security dialogue box. This problem turned out to be the most debilitating of all that BVI participants faced. Participants required sighted help to continue. The following is evidence of this problem as experienced by BVI3 and BVI5. The term "Jaws" referred to in this evidence is the screen-reader used by participants.

$\begin{array}{ll}\text { SR: } & \text { Moving to another question will save this response... go to previous } \\ & \text { question button... } \\ \text { BVI5: } & \text { Moving to another question will save this response, } \\ \text { SR: } & \text { Confirm question submission. Are you sure you want to ... Press space } \\ & \text { bar. } \\ \text { BVI5: } & \text { We are sure we want to go. So, I will press spacebar to continue. } \\ \text { SR } *: & \text { space. } \\ \text { SR: } & \text { Go to next question button. } 4 \% .84 \% . \\ \text { BVI5: } & \text { Waiting for. . } \\ \text { SR: } & \text { vertical bar. Go to last question button. Blank. vertical bar. Go to next } \\ & \text { question button. vertical bar. Go to next question button. vertical bar. } \\ & \text { Go to. vertical bar. Go to next question button. } \\ \text { SR*: } & \text { Enter. } \\ \text { SR: } & \text { Vertical bar. } \\ \text { BVI5: } & \text { Jaws is a bit slow right now. }\end{array}$


SR: $\quad$ 100\%. table, column0, row0. no button. more info button. To activate. Yes button. no button. to activate. Yes button. To activate. No button. to activate, press space bar. Yes button. To activate, press space bar.

BVI5: $\quad$ There's something to answer, yes or no. So, I will finally see if I can "Alt $B$ " it.

SR*: $\quad$ alt B. alt $B$.

BVI5: $\quad$ No. Can't read it to me. I have a question. There is a yes No question on my screen. What do I do?

Q: $\quad$ Click on yes

BVI5: $\quad$ I didn't know what was in that dialogue box.

SR*: $\quad$ Enter.

SR: $\quad$ Table, column18, row1.

BVI5: $\quad$ There apparently appeared a dialogue box that I could not read. But I had a yes button and a no button. So, I asked the instructor for directions.

BVI5 could negotiate the security information pop-up only with sighted intervention and avoided a more severe problem from inadvertently choosing "No". However, other participants ran into more severe problems. For example, we present verbal reports from BVI3's interaction below:

SR: $\quad$ Security information dialogue. To navigate use Tab...20\%

BVI3: $\quad$ Warning security.

SR: $\quad$ Thirty three percent

BVI3: $\quad H u h !$ This is weird.

SR: $\quad$ Retail Certificate Dialogue.

BVI3: $\quad$ This is a non-visual, this is a non-screen access thing. And I have no idea what it did. It is still loading the page. And, it wants me to do something. Do we have any visual assistance?

SR: $\quad$ Thirty two percent.

BVI3: $\quad$ I basically can't. . My screen...Jaws is not reading anything except for the progress bar announcements. And there's something else on this screen.

Q: $\quad$ Do you need help?

BVI3: $\quad$ You'd do better to do it with the mouse.

SR: $\quad$ Retailed certificate dialogue. To navigate, use tab.

BVI3: $\quad$ And that part is not accessible.

SR*: $\quad$ tab. Escape.

SR: $\quad$ Warning Security Dialogue.

SR*: $\quad$ Escape.

SR: $\quad$ Blackboard.

SR*: $\quad$ Alt Tab. Alt Tab.

SR: $\quad$ Blackboard Academic Suite. Microsoft internet explorer.

BVI3: $\quad$ Let's see what we did.

SR: $\quad$ No button, to activate. yes button, to activate. . Space bar.

BVI3: $\quad O h$ ! It's asking me a question. Hang on. Let me see what it wants.

SR*: $\quad$ Alt tab. Blackboard academic suite. Microsoft Internet Explorer. security information dialogue. This page contains both secure and nonsecure...

Do you want... .

BVI3: $\quad$ Yes we want to...

SR: $\quad \ldots$ (Silence) 
BVI3: $\quad$ Now, it basically just stopped.

$Q: \quad$ Where are you now?

BVI3: $\quad$ I have no idea.

SR: $\quad$ blackboard academic suite. Microsoft Internet Explorer.

BVI3: It says blackboard academic suite. But I'm seeing absolutely nothing. I need to get to question three. And right now, it's just, it's just not doing anything. So I'm gonna hit Alt Left Arrow. For some strange reason, I have a feeling. . Better yet, I'm going to hit the F5 key. It refreshes the screen....

SR: $\quad$...(Silence)

BVI3: $\quad$ And that didn't seem to work. So I'm going to hit Alt+Left Arrow. Takes us back.

SR: $\quad$ blackboard academic suite. Microsoft Internet Explorer.

SR*: $\quad$ Insert F7

SR: $\quad$ no links found.

BVI3: $\quad$ And that didn't seem to work either. So unfortunately, I have to close this out. But no worries, I can open it back. I will need sighted help to see what's going on. I'm stuck at some point. I don't know where I am. Nothing's found.

The security information pop-up remained on the screen and the screen-reader did not announce it or anything other than percentage values intermittently separated by unusually long pauses. A few moments later, it announced "Retail Certificate" dialogue which completely blocked the LMS from the screen-reader. BVI3 tried different problem-solving strategies to access the exam without luck. The "Retail Certificate" appears on choosing "No" in the security information dialogue box and renders the screen-reader speechless; a BVI user cannot access any exam-related information. We would like the reader to understand that when a screen-reader is speechless, the computer is unresponsive to the BVI user - a situation comparable to a "Blue Screen of Death" for a sighted user. The main culprit is the security information dialogue box that is not completely perceivable, understandable, or operable through a screen-reader. BVI students must spend additional time and effort trying to negotiate this dialogue. Our participants needed sighted assistance to move past this roadblock.

The security information pop-up problem involves two kinds of inconsistencies. There is inconsistency between expected and observed outcomes to confirm submission of exam answers. The expected outcome of this action is the navigation to a subsequent question page, however, the observed outcome of this action changes if the question is in essay-type format - it brings up a Security Information dialogue box. The second inconsistency is between the expected and observed outcomes of the action to dismiss the security information dialogue, which can be dismissed by a "Yes" or "No" in the dialogue box or through the escape key. The expected outcome is navigation to the next page, but the observed outcome changes: Choosing 'No' brings up a Retail Certificate and the Escape key does absolutely nothing. The inability to negotiate security information pop-up indicates gulfs of execution and evaluation. The gulf of execution is manifest in the failure to determine the correct action to dismiss the security information dialogue, and the gulf of evaluation is manifest in the inability to perceive the security information and difficulty understanding the "Yes" or the "No" buttons. This creates a serious roadblock for BVI students in completing exams online.

\section{Result of WCAG assessment of LMS design}

The inaccessible security information pop-up violates two web accessibility principles: the 'No Keyboard Trap' principle; and the Compatibility principle. 
Violation of the No Keyboard Trap principle: This principle, in WCAG's Success Criteria 2.1.2, requires that the keyboard focus should not be trapped by a Web page component. WCAG requires that Web sites allow users to move focus to and out of a component using the keyboard alone. WCAG requires the Web site to inform the user about the use of other standard exit methods unequivocally. Violation of this principle results in the Web content "trapping" the keyboard focus in Web page subsections. This was shown in the example discussed where a dialogue box trapped the keyboard focus and BVI participants could not negotiate the security dialog. WCAG notes that these dialogue boxes often trap keyboard focus and recommends informing users of the keystrokes to exit the dialogue box before the launch of the dialogue box and within the dialogue box. However, the LMS we examined provided no such instruction at any stage. Consequently, BVI participants were lost in how to dismiss the security dialog.

Violation of the Compatibility principle: Covered by WCAG Guideline 4.1, this principle requires maximizing compatibility of Web sites with current and future screen-reading technology and makes three important recommendations:

a. Avoid using poorly formed markup that breaks the screen-reader. The LMS in our analysis does not follow this recommendation where the dialog "broke" the screen-reader.

b. Avoid using unconventional markups that circumvent the screen-reader. The LMS in our analysis failed on this account since the Security Information dialogue box circumvented the screen-reader and rendered it speechless.

c. Expose information in the content following standard techniques for screen-reader operability. The LMS in our analysis did not comply with this recommendation. The screenreader could not recognize or interact with the information contained in either of the Security dialogs.

WCAG notes that screen-readers have difficulty keeping up with rapid advances in Web technology. When Web design follows conventions and maintains compatibility with standards, screenreaders can work with emerging technologies.

\section{Results of Web developer assessment of LMS design}

Web developer assessment identifies the source of the inaccessibility of security dialog as an alert dialogue box incompatible with screen-reading technology. Participants consistently attributed the use of an alert dialogue box by the LMS to inaccessibility and identified potential solutions.

"What happens here is that the system pops up a dialogue box that is not built in an accessible way. This pop-up is triggered by a Java applet. It is definitely possible to make this dialogue accessible. But obviously, not enough time was spent in analyzing this interaction between the Web page and the Java applet in triggering the pop-up."

"It is advisable that developers do not use the alert dialogue box. Instead, they should use the simple command mode as in a confirm dialogue box."

"Standard sites like Google and Microsoft allow the user to move between secured and unsecured sites in a minute. Basically, what happens is that the user gets only one confirmation dialogue that asks: "Moving from secure page to unsecure page. Do you want to go?" This is pretty simple."

The security dialog was an application side alert dialogue that blocks access to the essay-type question page and is incompatible with screen-readers. BVI students fail to perceive, understand, or operate on this information. They simply become helpless and get trapped within the box - use of alert dialogs makes aspects of LMS inaccessible and unusable for the BVI student. 
A feasible solution to address this problem is to use a confirm dialogue box to present security information. Screen-readers have easy access to information in a confirm dialogue box. Consequently, the information presented will be perceivable, understandable, and operable. BVI students can negotiate this content independently.

\section{Obscure Input Area for Essay-Type Question}

The input area assigned for essay-type questions is partially unavailable or obscured for BVI users. We explain the nature of this problem here.

\section{Results of BVI student assessment of LMS design}

Obscurity of essay-question input areas results in significant ambiguity and confusion for BVI users in answering the question. This ambiguity is multi-facetted and has three components:

a. Response method: BVI students cannot readily determine how to record their answer - pick an option or type a response;

b. Text formatting tools: Text formatting tools are not evident and BVI Students are disoriented in navigating them;

c. Difficulty locating text field: BVI students find it difficult, at times impossible, to locate the text box assigned for typing answer.

Evidence of this ambiguity is in verbal reports collected from every participant. We organize these verbal reports to highlight different aspects of the ambiguity.

\section{Ambiguity in the response method}

Verbal reports show participants' difficulty determining if the question requires choosing an option from a selection or typing out a response.

SR: $\quad$ Blank link save. Question. How would you define web accessibility?

BVI4: $\quad$ How would you define web accessibility?

SR: $\quad$ Graphic question three answers.

BVI4: $\quad$ So, I'm gonna hear the answers.

SR: $\quad$ Same page link graphic. skip visual text editors. link graphic. Edit. graphic text. Blank. blank.

BVI4: $\quad$ Oh no I can't find the answers. I'm gonna go up until it says something like answers...Maybe you have to type it? I'm gonna look and see if it says answers.

SR: $\quad$ Text blank link graphic blank same page link graphic question three answer blank how would you define blank click save find question three. Question completion status link blank blank table end.

BVI4: $\quad$ Maybe, you do have to type it because I'm not seeing anything that says answers. So, I should go back to where it says how would you define web accessibility?

SR: $\quad$ Five points link save blank how would you define web accessibility. Blank. graphic question three answers

BVI4: $\quad$ Oh! Ifound the answers. I'm gonna Enter on that.

SR: $\quad$ Enter. out of table. menu frame. Frame. visited link. announcements.

BVI4: $\quad$ Aww man! I can't find the answers. May be, I should look at the whole thing. So I'm gonna go down.

SR: $\quad$ Link external link link tools blank pause table link graphic communication collect graphic course .... 
BVI4: Gosh! Okay.

SR: $\quad$ Link graphic link table end link blank blank menu frame course content frame.

BVI4: $\quad$ I think I'm getting to it.

SR: $\quad$ Same page link e-learning visited link assignments link one web accessibility exercise... Graphic and...heading level one heading link.

BVI4: $\quad$ I'm going down.

SR: $\quad$ Blank table with two frames.

BVI4: $\quad$ Looking for the answers.

SR: $\quad$ Instructions this list contains... Blank five list end blank multiple, list of four test table.. Test table one blank blank... Blank table with graphic question.. Five points, how would you define. Blank graphic question three same page blank link graphic expanded blank text style.

BVI4: $\quad$ I think I did find the answers. Maybe not.

SR: $\quad$ Text style combo box one of eight blank font size.

BVI4: $\quad I$ think expanded is one of the answers.

SR: $\quad$ Blank text text blank.

BVI4: $\quad$ It just sounded different. May be, I went to something wrong. Well, I thought I messed up on it. I'm going up to where it says expanded.

SR: $\quad$ Graphic expanded.

BVI4: $\quad$ It's one of the answers I think. I'm gonna Enter on it.

SR: $\quad$ Answer table column one row three expanded visited link graphic.

BVI4: Gosh!

BVI4 could not determine how to appropriately respond in the essay-type question. The screenreader announced a page object as "Graphic Link Question 3 Answers". This created an expectation that multiple answer choices of a question lay ahead. The link "Expand" were particularly distractive for participants; one user mistook it as a possible answer while another expected an input field. These contributed to confusion about the appropriate response.

\section{Ambiguity about text formatting tools}

Verbal reports show participants' difficulty perceiving the affordances of formatting tools in answering questions in the LMS. Participants were disoriented in navigating the tools. To a screenreader, the formatting tools appear interspersed in the question text and the edit box. Poorly labeled tools disoriented students, losing them in unfamiliar interface objects.
BVI1: $\quad \quad \quad \quad$ Now I answer question three. So, now I'm going to try to...
SR: $\quad$ Question three. Five points, how will you define web accessibility. Blank. Graphic question three answers. Expand. . Text style. Same page link graphic. Italic. same.. fonts. formats....
BVII: $\quad$ Down arrow, down arrow.
SR: $\quad$ Same page link graphic align left...click submit to submit this assessment.
BVI1: I Ion't understand what this is. I don't really understand what's going on with this part of the question, with the Internet. I don't understand why it's saying a whole bunch of superscript, numbering, bullets, indents, it's kind of tough. It's not really telling me. I mean, it's far more easier to do like radio buttons when it came to like five out of four when it was like multiple choice that way. Otherwise, if it's this way it's harder, it's much more tough.
Q: $\quad$ How did you know that it is not a multiple choice question? 
BVI1: $\quad$ Because it didn't say... because it said multiple attempts on the first question. But I don't really know how to answer these. I don't understand how to really answer them.

SR: $\quad$ Question 3 text question 3, 5 points.

BVII: $\quad$ Oh! It's text questions. You have to write your answers into it. Got it.

The evidence highlights the ambiguity of BVI1 regarding the purpose of text formatting controls below the question text. He could not perceive the affordances of these objects in the context of answering the question. He came across a link labeled "Skip Visual Text Edit Buttons." He was unable to make the connection between the link and its purpose - jump over these controls to the edit box. All this contributed to the ambiguity about the text formatting tools.

\section{Difficulty locating text field}

Verbal reports show participants' difficulty in finding the text box to type their answers to the essay-type question.

SR: $\quad$ Five points. how will you define web accessibility?

BVI2: $\quad H o w$ would I define web accessibility? Keeping in my mind, I am going to go down the edit field and type the answer.

SR: $\quad$ Blank. Text style. Same page link graphic italic, same.. fonts, formats.... .. Same page link graphic. Align left...Click submit to submit this assessment

BVI2: $\quad$ I am having difficulty. I need to enter into the edit field and answer the question. But it's not reading the edit field. It's reading the forms mode, but not reading the edit field. I'm on the webpage and I can't seem to find the edit field.

SR: $\quad$ Collapse. Frame. Blank. Frame end.

BVI2: $\quad$ Last time between the frame and frame end there was the edit box where I could type the answer in; now it is not. It just says blank. Don't know what to do.

BVI2 could not locate the edit box to type his response - the difficulty was insurmountable. Unlike the sighted, a BVI student cannot instantly recognize that a question requires typing a response from quick visual scans. The screen-reader announcements were interpreted by the BVI student as a place to type something as evidenced in the verbal reports:

Q: $\quad$ Can you tell how you concluded that this question required you to type an answer considering you were unsure a few minutes back?

BVI1: $\quad$ What I did was I figured, because I read the beginning of the question before and it said text style which I thought write your answer. And when it said "Edit," "expanded," something, "links," and then I pressed the up arrow and it said "Edit," I just wrote my response to that question.

In the essay question, the LMS organizes relevant components in this order: instruction; question text; a bunch of interface objects (e.g., text formatting tools, etc.); edit box; and other interface objects (e.g., link Expand, Collapse, etc). A BVI student tries to locate the screen-reader announcement "Edit" in the middle of a long announcement stream of labels of surrounding objects. Locating the edit box was a significant challenge for participants. BVI participants spent extra time and effort due to this ambiguity and some could not continue with the online exam.

Systems interaction problems indicate a gulf between the user's cognition of the system and the actual behavior of the system. According to this tenet, ambiguity in answering essay questions 
involves discrepancies between the expected and observed outcomes of the action. The screenreader reads information sequentially from top left to bottom right and the user has access to only a small chunk of this information at a given time. They do not hear the edit box in the page. Instead, they hear individual formatting tools on multiple lines. According to the Action Model (Norman, 1988), ambiguity in essay-type question pages indicates a gulf of evaluation that manifests as difficulty to determine a response, difficulty to perceive affordances of text formatting, and difficulty to understand that the edit box lies beyond these tools.

The expected outcome of Confirming Question Submission is navigation, while the observed outcome for BVI students' changes; for if the subsequent question is in essay-type format it brings up a Security Information dialogue box.

\section{Result of WCAG assessment of LMS design}

Our analysis explains that the problem of an obscured input area in LMS for essay-type questions represents a violation of six design principles on Web accessibility or usability. These principles include:

- Sensory Characteristic principle of Web accessibility;

- Link Purpose principle of Web accessibility;

- Name, Role, Value principle of Web accessibility;

- Visibility principle of Web usability;

- Learnability and Efficiency principles of Web usability;

- Good Mapping principle of Web usability

In the following, we discuss how the LMS design violates each of these principles.

Violation of the Sensory Characteristic principle: WCAG's Success Criterion 1.3.3 requires that instructions to help users understand and operate Web content should not rely exclusively on sensory characteristics of an object such as visual location or orientation. WCAG recommends providing additional instruction so users do not lose information due to inaccessible formats. The LMS in our analysis relies on visual location and orientation of interface objects to communicate information related to the response method and the utility of text formatting tools for essay-type questions. It did not include any instruction text informing students about the availability of the input field beyond the question text and the text formatting toolbar.

Violation of the Link Purpose principle: WCAG's Success Criterion 2.4.4 asserts "The purpose of each link can be determined from the link text alone or from the link text together with its programmatically determined link context." The intent is to help users understand the utility of a link. Our BVI participants could not understand the purpose of many links on the essay-type question page. Noteworthy is the link labeled "Expand;" BVI participants were not able to understand its purpose. The poor labeling and its position below the question text were misleading for the BVI students.

Violation of the Name, Role, Value principle: WCAG's Success Criterion 4.1.2 requires that names and roles of all interface objects should be programmatically determined. It further requires that states, properties, and values that can be set by the user should be programmatically set. It requires that notification of changes to these items be available to the screen-reader to ensure that the screen-reader can access information pertaining on the state of interface objects in a Web page. In our analysis, the input area in the essay-type question page did not have a name or a label describing the purpose. There was no label whatsoever, explicit or implicit, that the screenreaders employed that participants could access. 
Violation of the Visibility principle: Norman (2002) requires Web design to help users readily perceive the state of a Web site and derive action alternatives by just observing. In our analysis BVI participants could not find the text box for the essay-type question.

Violation of the Learnability and Efficiency principles: These principles of usability discussed in Nielsen (1993) are interrelated. The Learnability principle helps make first-time users productive and efficient. The Efficiency principle helps users accomplish Web interaction tasks quickly without spending much cognitive effort once they have learned the Web site. Our analysis shows that BVI participants spent a lot of time and effort locating the input field and understanding the utility of the text formatting controls. This had adversely affected their productivity and BVI users often required sighted intervention.

Violation of the Good mapping principle: Norman (2002) requires that Web design help users determine the relationships between actions and results, between the controls and their effects, and between the system state and what is visible. In our analysis BVI participants were unable to determine the relationship between the text formatting tools and their effect. They could not understand the consequences of activating links.

\section{Results of Web developer assessment of LMS design}

Our analysis of Web developer assessment informs that the source of the obscurity of the input area for essay-type question problems is the poor labeling convention for three interface objects:

a. A graphic "Question Three Answers" that created confusion in the appropriate response;

b. A text formatting toolbar that created confusion about formatting controls; and

c. An edit box where it was difficult to locate the text area for typing an answer.

Participants identified a graphic right below the question text that was assigned a misleading label. The label "Graphic Question Three Answers" created a false expectation about possible answers. Participants identified a text formatting toolbar without a label. They explained that this confuses screen-reader users leading to disorientation. Participants pointed out that the text area did not have a descriptive caption. Evidence of this explanation in the form of summarized responses of participants is provided:

"We need information before we enter the text area field. This information is not available here. And that particular graphic that says "Question 3 answers" is misleading."

Responding to the question about the problem source, WD3 identified the misleading graphic, and suggested improvements in the LMS design as follows:

"Instead of saying Question 3 Answer, it could say "Space to enter answer for question 3". So, that could be just a question of modifying that label to be more descriptive."

Recognizing a lack of clear guidance for screen-reader users about the response, WD5 observed:

"One solution may be to add in a description of what's going to come in immediately as you are pulling up a text area. It's definitely possible to ensure that for the text area, you insert a short description that is only visible to the screen reader."

About the disorientating effect of the poorly labeled text formatting toolbar, WD1 observed: 
"You are provided a space to enter a long form answer. And that space includes a toolbar that has buttons which allow you to format the text you are entering. It is confusing for a screen-reader user."

Further elaborating on this point, WD2 explained its negative implications for the BVI user as follows:

"These are all text formatting options that the user is not supposed to read. This is the first mistake. If there were only 5 to 6 options, then the user could have been able to make out where the screen-reader announced "Edit". Because there are thirty to forty announcements that correspond to specific options, the user gets confused."

WD2 further explained:

"I think that HTML syntax must not be put into readable content. The 2nd thing is that the design should be in a way that the contents are grouped correctly; users must know which section they are going into so that they are able to make the decision whether to go or not to go."

On the topic of a text area with no descriptive caption, participant WD1 explained:

"The text area currently doesn't have a caption associated with it. For any text box or input control they say that you should put the caption or label."

On this same topic, participant WD2 said:

"Here the problem is the developer has not surrounded the input attribute with a label."

WD4 discussed the problem of caption-less text area as follows:

"If there is a question, it should be provided with some label saying this is Question one. If there is an input text box, we must define it by saying that this particular text box is being used for answering question number one. That will be very informative for the user."

The source of the obscured input area is a lack of appropriate labels for three interface objects relevant to an essay-type question: a graphic, a set of formatting tools, and an edit box. Remedial measures to potentially address this include:

(1) Replace "Question Three Answers" with "Space to Type Question Three Answer";

(2) Provide a label for the text formatting toolbar to tell the user about the edit box; and

(3) Include a meaningful caption such as "Type your answer for question 3 here".

It should be noted that these are suggestions offered by developers and not design principles. They have the potential to remove the ambiguity problem and are worth further investigation and verification.

\section{Practical Implications}

Results demonstrate the efficacy and feasibility of the TUME technique in providing more complete, practically-relevant, solution-oriented assessment of LMS accessibility and usability as a test-taking tool for BVI students. The three question formats used are also common in Web-based 
surveys, online job applications, and online college applications. In addition, they represent the standard forms of information input for web forms. Therefore, while the context of the study was a particular LMS, the usability issues that BVI students encountered here are conceivably common to other forms of entering information on the web.

Our findings have significant implications for BVI e-learning, educators, and learning technologists. BVI students currently face significant challenges due to a lack of accessibility and usability of e-learning tools. The TUME technique can help create more accessible and usable elearning environments where BVI students can function and learn effectively.

Academic institutions are required by laws on equal Web access, such as the Americans with Disabilities Act and Section 508 of the Rehabilitation Act, to make their e-learning implementations accessible to students with disabilities. While existing evaluation techniques could possibly aid in providing technical accessibility, they do not ensure effective access for such students. The TUME technique can help academic institutions avoid litigation by assessing the effective accessibility of e-learning tools for BVI students. Our future research will investigate if the TUME technique is extendible to students with other disabilities.

Web developers, including learning technologists, are unaware of BVI students' unique Web accessibility and usability needs. Design standards such as the Web Content Accessibility Guidelines are good starting points, but they are difficult to comprehend and implement (Clark, 2006). The TUME technique provides actionable guidance in creating more accessible and usable elearning environments for the BVI user. The solutions identified by TUME are feasible to implement since they consider the technical feasibility perspectives of Web developers.

With further validation and generalization across more problems, the evaluations presented here can provide the basis for developing design principles that lead to accessible, usable, and useful LMS for BVI students. Such design principles can form the basis for automation of Web accessibility and usability evaluation for non-visual interaction (NVI). NVI assumes significance in mobile and ubiquitous learning environments, as well as in multi-tasking situations.

\section{Conclusion}

This paper presented a novel task-oriented, user-centered, multi-method evaluation (TUME) technique for a holistic, practically-relevant, solution-oriented assessment of the accessibility, usability, and utility of a Web-based application. A Web-based task is the unit of analysis for this evaluation. This is more useful than traditional evaluation methods that perform an interfaceelement-wise assessment of accessibility and usability. Using the task context of an online exam, the paper demonstrated the utility of the TUME technique to evaluate a learning management system (LMS) for blind and visually impaired (BVI) users. This evaluation synthesized the results of:

(1) BVI student assessment using verbal protocol analysis;

(2) WCAG assessment using text analysis;

(3) Web developer assessment using structured, open-ended interviews.

It showed how to identify design errors in a LMS task environment, the consequent problems (challenges and roadblocks) BVI students face in completing this task, the technical sources of these problems, and remedial measures to potentially improve LMS utility as a test-taking tool for the BVI student. This provides a more complete, practical, and solution-oriented approach to accessibility and usability evaluation. Completeness is achieved through synthesis of the viewpoints of the user, Web accessibility and usability standards, and the Web developer - three key entities in Web accessibility and usability. The practical utility comes from its task-oriented nature that 
places accessibility and usability of a Web-based application in the context of a task it was designed to support. The solution-oriented aspect is that it provides actionable guidance on addressing a problem and not just identifying it. Such evaluation will help academic institutions enhance the success of their e-learning implementations by accommodating students for whom non-visual interaction is a necessity. Currently, we are conducting a large-scale evaluation of commonly used e-learning tools to identify a comprehensive set of accessibility and usability problems for BVI students and other groups who use NVI.

The TUME technique is useful to make conjectures about design modifications that can potentially meet the accessibility and usability needs of BVI users in a Web interaction task. Such conjectures must undergo a validation process before becoming task-specific design principles on accessibility and usability for Web-based applications. These design principles guide the development of IT artifacts to effectively improve accessibility and usability. As this paper shows, the solution can be simple design modifications to the LMS interface, which can be achieved at a reasonable cost to the developer. For instance, modifying the design of an essay-type question page to include a short message underneath the question text or inserting a caption for the input area can be simple adjustments for the developer. However, these adjustments help improve the visibility of the input area and reduce ambiguity and disorientation for BVI students. Potential design solutions such as those identified in this paper are under evaluation in our on-going research employing an experimental design with BVI students and using a prototype online exam interface.

The Americans with Disabilities Act, Section 508 of the Rehabilitation Act, and Individuals with Disabilities \& Education Act require that learning tools such as LMS are accessible and usable to BVI students. In this backdrop, our finding that a commonly used LMS lacks accessibility and usability assumes significance for academic institutions and education technologists. Our multimethod evaluation can help the industry and academia feasibly meet their social, moral, and legal obligations by ensuring the accessibility and usability of LMSs and other Web applications.

TUME has value beyond LMS and BVI users. It can be used to test the accessibility and usability of specific task environments offered by website genres other than LMS, including social media, healthcare portals, online stores, and electronic libraries. It provides improved accessibility and usability evaluation for non-visual interaction. NVI is needed in mobile and ubiquitous computing environments that have little to no displays. NVI assumes significance in multi-tasking situations where one task demands complete visual attention, for example a motorist reading directions while focusing on the road ahead. The TUME approach is extensible to other user types including both typical and atypical groups. For example, it may be feasibly adapted to test LMS accessibility, usability, and utility for older adults with limited dexterities. In summary, TUME has value for the evaluation of systems accessibility and usability in general.

\section{References}

Alavi, M., \& Gallupe, B. R. (2003). Using information technology in learning: Case studies in business and management education programs. Academy of Management Learning \& Education, 2(2), 139-153.

American Foundation for the Blind. (2008). Facts and figures on Americans with vision loss. Retrieved from http://www.afb.org/Section.asp?SectionID=15\&DocumentID=4398

Armstrong, H. L. (2009). Advanced IT education for the vision impaired via e-learning. Journal of Information Technology Education: Research, 8, 243-257. Retrieved from http://www.jite.org/documents/Vol8/JITEv8p243-256Armstrong685.pdf

Arroway, P., Davenport, E., Xu, G., \& Updegrove, D. (2010). Educause core data service. Fiscal year 2009 summary report. Boulder, $\mathrm{CO}$ : Educause. 
Babu, R. (2011). Developing an understanding of the accessibility and usability problems blind students face in web-enhanced instruction environments. (Doctoral Dissertation). Retrieved from ProQuest Dissertations and Theses. (Accession Order No. AAT 3473492)

Babu, R., \& Singh, R. (2009). Evaluation of Web accessibility and usability from blind users' perspective: The context of online assessment. Proceedings of The Fifteenth Americas Conference on Information Systems. San Francisco, CA: ACM.

Babu, R., Singh, R., \& Ganesh, J. (2010). Understanding blind users' Web accessibility and usability problems. AIS Transactions on Human Computer Interaction, 2(3), 78-91.

Babu, R., Singh, R., Iyer, L.S., \& Midha, V. (2007). Differences in factors affecting academic success for disabled individuals in technology-mediated learning environments. Proceedings from AMCIS 2009: Thirteenth Americas Conference on Information Systems. Keystone, CO.

Brophy, P., \& Craven, J. (2007). Web accessibility. Library Trends, 55(4), 950-972.

Brown, L. M., \& Kaaresoja, T. (2006, April). Feel who's talking: Using tactons for mobile phone alerts. In CHI '06 Extended Abstracts on Human Factors in Computing Systems (pp. 604-609). New York, NY: ACM.

Christian, K., Kules, B., Shneiderman, B., \& Youssef, A. (2000). A comparison of voice controlled and mouse controlled web browsing. Proceedings of the Fourth International ACM Conference on Assistive Technologies, Arlington, VA (pp. 72-79). New York, NY: ACM.

Clark, J. (2006). To hell with WCAG 2. A List Apart. Retrieved from http://www.alistapart.com/articles/tohellwithwcag2

Correani, F., Leporini, B., \& Paternò, F. (2004). Supporting usability for vision impaired users in web navigation. User-Centered Interaction Paradigms for Universal Access in the Information Society: Eighth ERCIM Workshop on User Interfaces for All, Vienna, Austria. 242-253.

Cotton, D., \& Gresty, K. (2006). Reflecting on the think-aloud method for evaluating E-learning. British Journal of Educational Technology, 37(1), 45-54.

Crabtree, B. F., \& Miller, W. L. (Eds). (1999). Doing qualitative research in primary care: Multiple strategies (2nd ed.). Newbury Park, CA: Sage Publications.

De Smet, C., Bourgonjon, J., De Wever, B., Schellens, T., \& Valcke, M. (2012). Researching instructional use and the technology acceptation of learning management systems by secondary school teachers. Computers \& Education, 58, 688-696.

Di Blas, N., Paolini, P., \& Speroni, M. (2004, June). Usable accessibility to the web for blind users. Proceedings of the 8th ERCIM Workshop: User Interfaces for All. Vienna, Austria.

Ericsson, K. A., \& Simon, H. A. (1993). Protocol analysis: Verbal reports as data. London: MIT Press.

Ericsson, K. A., \& Simon, H. A. (1984). Protocol analysis: Verbal reports as data (2nd ed.). Cambridge, MA: MIT Press.

Freire, A. P., Goularte, R., \& Fortes, R. P. (2007, October). Techniques for developing more accessible web applications: A survey towards a process classification. Proceedings from the $25^{\text {th }}$ Annual ACM International Conference on Design of Communication (pp. 162-169). El Paso, TX: ACM.

Greenbaum, J., \& Kyng, M. (1991). Design at work: Cooperative design of computer systems. Philadelphia, PA: Psychology Press.

Goodhue, D. L. (1986). IS attitudes: Towards theoretical definition and measurement clarity. Proceedings from the Seventh International Conference on Information Systems, San Diego (pp. 181-194).

Goodwin, N. (1987). Functionality and usability. Communications of the ACM, 30(3), 229-233.

Hamuy, E., \& Galaz, M. (2010). Information versus communication in course management system participation. Computers \& Education, 54, 169-177. 
Heim, S. (2007). The resonant interface: HCI foundations for interaction design. Boston, MA: AddisonWesley Longman Publishing.

Jana, R. (2009, September 23). How tech for the disabled is going mainstream. Businessweek. Retrieved from: http://www.businessweek.com/stories/2009-09-23/how-tech-for-the-disabled-is-goingmainstream

Katz-Haas, R. (1998). Ten guidelines for user-centered web design. Usability Interface, 5(1).

Kelly, B., Sloan, D., Phipps, L., Petrie, H., \& Hamilton, F. (2005). Forcing standardization or accommodating diversity? A framework for applying the WCAG in the real world. Proceedings from the 2005 International Cross-Disciplinary Workshop on Web Accessibility, Chiba, Japan (pp. 46-54).

Kim-Rupnow, W. S., \& Burgstahler, S. (2004). Perceptions of students with disabilities regarding the value of technology-based support activities on postsecondary education and employment. Journal of Special Education \& Technology, 19(2), 43-56.

King, M., Thatcher, J. W., \& Easton, R. (2005). Managing usability for people with disabilities in a large Web presence. IBM Systems Journal, 44(3), 519-535.

Krug, S. (2005). Don't make me think: A common sense approach to Web usability (2nd ed.). Thousand Oaks, CA: New Riders Publishing.

Lazar, J., Allen, A., Kleinman, J., \& Malarkey, C. (2007). What frustrates screen reader users on the Web: A study of 100 blind users. International Journal of Human-Computer Interaction, 22(3), 247-269.

Leuthold, S., Bargas-Avila, J. A., \& Opwis, K. (2008). Beyond Web content accessibility guidelines: Design of enhanced text user interfaces for blind internet users. International Journal of HumanComputer Studies, 66(4), 257-270.

Lonn, S., \& Teasley, S. D. (2009). Saving time or innovating practice: Investigating perceptions and uses of learning management systems. Computers \& Education, 53, 686-694.

Lumsden, J., \& Brewster, S. (2003, October). A paradigm shift: Alternative interaction techniques for use with mobile \& wearable Devices. Proceedings from the Thirteenth Annual IBM Centers for Advanced Studies Conference (CASCON'03), Markham, Ontario, Canada.

Mankoff, J., Fait, H., \& Tran, T. (2005, April). Is your Web page accessible? A comparative study of methods for assessing Web page accessibility for the blind. Proceedings of the SIGCHI Conference on Human Factors in Computing Systems, 41-50. ACM.

McCormick, J. (2000). The new school. Newsweek, 135(17), 60-62.

Millar, S. (1994). Understanding and representing space: Theory and evidence from studies with blind and sighted children. Oxford: Oxford University Press.

Moss, T. (2006). The new W3C accessibility guidelines evaluated. Retrieved from http:/www.webcredible.co.uk/user-friendlyresources/web-accessibility/wcag-guidelines-20.shtml

Murray, M., Perez, J., Geist, D., \& Hedrick, A. (2012). Student interaction with online course content: Build it and they might come. Journal of Information Technology Education: Research, 11, 125-140. Retrieved from http://www.jite.org/documents/Vol11/JITEv11p125-140Murray1095.pdf

Neumeier, P. (2005). A closer look at blended learning: Parameters for designing a blended learning environment for language teaching and learning. Recall, 17(2), 163-178.

Newell, A., \& Simon, H.A. (1972). Human problem solving. Englewood Cliffs, NJ, Prentice-Hall.

Nielsen, J. (1993). Usability engineering. San Diego, CA, Morgan Kaufmann.

Norman, D. A. (1988). The design of everyday things. New York: Basic Books.

Norman, D. A. (2002). The design of everyday things (2nd ed.). New York: Basic Books

Pituch, K. A., \& Lee, Y. K. (2006). The influence of system characteristics on E-learning use. Computers \& Education, 47, 222-244. 
Pynoo, B., Devolder, P., Tondeur, J., van Braak, J., Duyck, W., \& Duyck, P. (2011). Predicting secondary school teachers' acceptance and use of a digital learning environment: a cross-sectional study. Computers in Human Behavior, 27, 568-575.

Schuler, D., \& Namioka, A. (1993). Participatory design: Principles and practice. Hillsdale, NJ: Lawrence Earlbaum Associates.

Shneiderman, B., \& Plaisant, C. (2004). Designing the user interface. Reading, MA: Addison-Wesley.

Simon, B., \& Cutts, Q. (2012). Peer instruction: A teaching method to foster deep understanding. Communications of the ACM, 55(2), 27-29.

Sun, P. C., Tsai, R. J., Finger, G., Chen, Y. Y., \& Yeh, D. (2008). What drives a successful E-learning? An empirical investigation of the critical factors influencing learner satisfaction. Computers \& Education, 50, 1183-1202.

Theofanos, M. F., \& Redish, J. (2003). Guidelines for accessible and usable Web sites: Observing users who work with screen readers. Interactions, 10(6), 38-51.

Thinus-Blanc, C., \& Gaunet, F. (1997). Representation of space in blind persons: Vision as a spatial sense? Psychological Bulletin, 121, 20-42.

Todd, P., \& Benbasat, I. (1987). Process tracing methods in decision support systems: Exploring the black box. Management Information Systems Quarterly, 11(4), 493-512.

Van Raaij, E., \& Schepers, J. (2008). The acceptance and use of a virtual learning environment in China. Computers \& Education, 50, 838-852.

\section{Acknowledgements}

This article is based on our work supported by the National Science Foundation under Award No. BCS-0938539. Another part of this work was supported by the Triad Interuniversity Planning Project Grant under Fund No. 225285.

\section{Biographies}

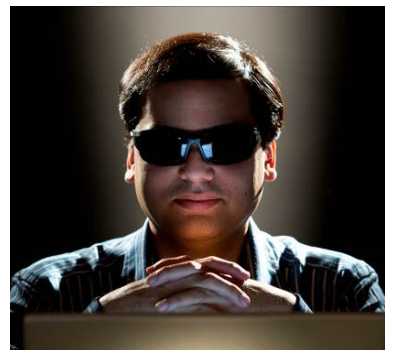

Rakesh Babu is an assistant professor in the School of Information Studies at the University of Wisconsin-Milwaukee. He is blind and has strong motivation for research topics relevant to empowering the blind in the information society. He leads an interuniversity research team to establish a research center with this objective. His research expertise includes Web accessibility and usability, Human-Centered Computing, Cognitive Modelling, Online Education and e-Healthcare. He has multiple prior and on-going research projects sponcered by National Science Foundation, European Research Council, and Research Council of Norway. His research appears in multiple journals and conference proceedings. He is a regular reviewer for multiple journals and international conferences.

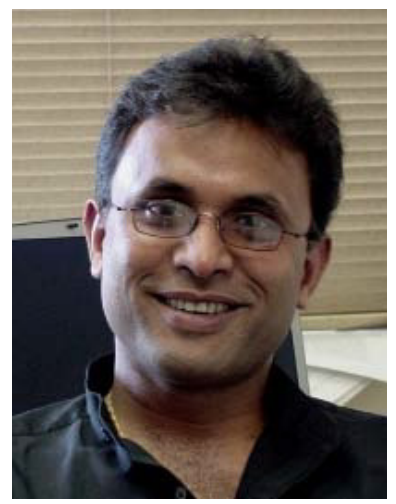

Rahul Singh is an Associate Professor in the Department of Information Systems and Supply Chain Management, Bryan School of Business and Economics at The University of North Carolina at Greensboro. He obtained his Ph.D. in Business from Virginia Commonwealth University. His research interests include systems accessibility and usability, on-line education, Systems Security, Secure Business Process Design, Knowledge Management and Business Intelligence. His research work is published in leading journals including Journal of AIS 
(JAIS), IEEE Transactions on Systems Man and Cybernetics, AIS Transactions on Human Computer Interaction, Communications of the ACM, European Journal of Information Systems. He is the Editor-In-Chief for the Journal of Information Science and Technology (JIST). He is a member of the editorial board for the International Journal of Semantic Web and Information Systems, the International Journal for Intelligent Information Technologies, the Journal of Information Technology Theory and Applications, and the International Journal of Information Security and Privacy. 